\title{
A Cross-Sectional Study on Balance Deficits and Gait Deviations in COPD Patients
}

\author{
Priyanka Jirange, ${ }^{1}$ K. Vaishali $\left(\mathbb{D},{ }^{1}\right.$ Mukesh Kumar Sinha, ${ }^{1}$ \\ Kalyana Chakravarthy Bairapareddy, ${ }^{2}$ and Gopala Krishna Alaparthi $\mathbb{D}^{2}$ \\ ${ }^{1}$ Department of Physiotherapy, Manipal College of Health Professions, Manipal Academy of Higher Education, Manipal 576104, \\ Karnataka, India \\ ${ }^{2}$ Department of Physiotherapy, College of Health Sciences, University of Sharjah, Sharjah, UAE
}

Correspondence should be addressed to K. Vaishali; vaishali.kh@manipal.edu

Received 6 November 2020; Accepted 18 December 2020; Published 6 January 2021

Academic Editor: Andrea S. Melani

Copyright (c) 2021 Priyanka Jirange et al. This is an open access article distributed under the Creative Commons Attribution License, which permits unrestricted use, distribution, and reproduction in any medium, provided the original work is properly cited.

\begin{abstract}
Background. The gait abnormalities were linked to the balance deficits in the previous studies. However, the deviations in the gait parameters in COPD are currently not known. The study aims to compare gait parameters, static and dynamic balance, and risk of falls in COPD with those in non-COPD individuals. Method. Fourty-two patients with COPD aged 45 years and gender-matched control subjects were included in the study. Gait parameters were assessed by Win-Track gait analyzer, the static balance was assessed by posturography, and the dynamic balance was assessed by the time up and go test. The fear of falls was assessed by Falls Efficacy Scale. Results. COPD individuals had decreased static and dynamic balance as assessed by posturography $(p<0.05)$ and TUG $(p<0.01)$, respectively. A significant difference in swing duration $(p=0.004)$ and also increased risk of falls $(p<0.01)$ was observed in COPD patients as compared to non-COPD individuals. Conclusion. COPD individuals have increased swing duration, reduced static and dynamic balance, and increased fear of falls as compared to non-COPD individuals.
\end{abstract}

\section{Introduction}

COPD is forecast to become the world's third major cause of death in the global disease burden by $2030[1,2]$. The 2016 Global Disease Burden Study reports a prevalence of 251 million COPD instances worldwide [3]. According to a 2006 multicentre research sponsored by the Indian Medical Research Council (ICMR), the prevalence of COPD in people over 35 years in India is 4.1 percent [4].

Chronic obstructive pulmonary disease (COPD) is a preventable and treatable disease with several extrapulmonary manifestations, including cachexia and skeletal muscle dysfunction, contributing to morbidity and mortality $[5,6]$. It has been well established that skeletal muscle function (strength and endurance) and structure (fiber size, fiber-type distribution, capillary density, and metabolic capacity) are altered in patients with COPD, leading to impaired balance and mobility [6,7]. Balance and mobility are significant aspects of most daily living activities, and studies have shown that decreased muscle power and hypoxia affect static and dynamic balance in COPD patients $[8,9]$. Balance impairment is associated with an increased risk of falling with a rise in adult morbidity rate [10]. A cross-sectional study revealed that $\mathrm{COPD}$ is the most prevalent chronic condition with the highest prevalence of falls, second only to osteoarthritis [11].

In COPD patients, reduced lower limb function and balance deficit may compromise the stability needed for walking, thus altering gait in COPD patients compared to healthy elderly patients $[12,13]$. However, the variations in the spatiotemporal gait parameters in COPD patients are presently not known. The objective of the study was to compare gait parameters, static and dynamic balance, and risk of falls in COPD with those in non-COPD individuals. 


\section{Methods}

The study protocol has been evaluated and endorsed by the institutional research committee and university ethical committee. All subjects were notified of the study's purpose and procedure. In a cross-sectional study, 42 patients aged 45 years and diagnosed with COPD and gender-matched individuals with no history of COPD (non-COPD) were recruited. Patients between 50 and 60 years of age with medically stable COPD were categorized according to GOLD criteria. The research respondents who had history of orthopaedic, neurology, central or peripheral vestibular illnesses, and diabetic neuropathy were excluded. Age- and gender-matched healthy subjects were recruited during the camps as part of the community screening. Individuals who were willing to participate and gave written informed consent were part of the study. For all respondents, weight, height, and body mass index were recorded as part of the initial assessment. COPD patients were tested for pulmonary function, and the severity of the disease was investigated.

\section{Gait Evaluation}

Using the Win-Track gait analyser, gait parameters were evaluated. It comprises a $1616 \mathrm{~mm}$ long platform with a width of $652 \mathrm{~mm}$ and a height of $30 \mathrm{~mm}$ [14]. Three trails were given to all the participants and were instructed to walk on the platform with at least three steps. Three-step gait protocol was used as it had shown good reliability in measuring temporal variables [14]. Subjects were advised to look forward and walk on the platform at a comfortable pace. The data were uploaded to a computer for further quantitative analysis.

\section{Balance Assessment}

The assessment of static balance was done by posturography (Good BalanceTM, Metitur Ltd., Finland) [15, 16]. The participants were made to stand on the platform of posturography and look for 30 seconds at a fixed point on the opposite wall. For the eye-open on foam (EOF) and eyeclosed on foam (ECF) conditions, the participants stood on a $2.5 \mathrm{~cm}$ thick piece of foam placed on the top of the force plate. The postural sway was observed with eyes open and closed on stable surfaces on the force platform in normal standing.

\section{Timed Up and Go (TUG) Test}

To evaluate the dynamic balance, the TUG test was performed. For the evaluation, a chair with armrest, a stopwatch, and an inch tape were used to mark the distance of three meters. A practice session was conducted before the timed test. The participants sat down and were requested to stand up, walk three meters, turn around, walk back to the chair, and sit down. The time taken to complete the test was recorded, and the 14-second cutoff value was used to determine the risk of falling $[17,18]$.
TABle 1: Demographic data of the subjects.

\begin{tabular}{lccc}
\hline & COPD & Non-COPD Subjects & $p$ value \\
\hline$N$ & 42 & 45 & \\
Age (mean \pm S.D.) & $61.90 \pm 4.61$ & $60.47 \pm 6.18$ & 0.16 \\
BMI (mean \pm S.D.) & $22.22 \pm 3.31$ & $23.50 \pm 3.27$ & 0.11 \\
Lung function & & & \\
FEV 1 (\% & $37.30 \pm 7.99$ & - & \\
predicted) & & - & \\
FVC (\% predicted) & $60.07 \pm 10.58$ & - & \\
FEV1/FVC & $61.30 \pm 10.39$ & - & \\
\hline
\end{tabular}

COPD: chronic obstructive pulmonary disease.

\section{Fear of Falls Assessment}

Falls Efficacy Scale (FES) questionnaire was used to evaluate the fear of falling or continuous worry about falling that may restrict daily living activities. The participants were asked to rate on a four-point Likert scale about the possibility of falling while performing 16 different activities. A high score on the total score of 28-64 in FES-I scale (PRoFaNE group) indicates the high concern about falling [19].

\section{Data Analysis}

Data were analysed using SPSS version 15 . Test for normality was done. Descriptive statistics were used to analyse the demographic data. Independent $t$-test was used for the comparison of demographic data. Mann-Whitney U-test was used to compare the gait parameters, posturography values, scores of Falls Efficacy Scale, and time of TUG test between COPD and healthy subjects. Spearman's rank correlation coefficient was used to determine the correlation between Fev1\% and balance parameter for COPD participants. The level of significance was set at $p<0.05$.

\section{Results}

The static balance, gait assessment and TUG tests were successfully performed by both groups of people without losing balance. The body mass index was comparable between the two groups. The characteristics of the subjects are presented in Table 1. No significant difference was observed in any demographic variable. COPD individuals have increased swing duration in comparison with non-COPD individuals with $p<0.05$ shown in Table 2 . The findings of both COPD and non-COPD posturography measures are summarised in Table 3. In contrast to non-COPD individuals, all posturographic variables indicating more postural sway in COPD individuals, however, NSEC-AP, ML sway, and NS EO-AP sway are statistically significant $(p<0.05)$. In addition, the velocity moment was found to be more in COPD individuals compared to non-COPD individuals, although NSEO-VM is statistically significant $(p<0.05)$. The outcome of the FES-I score is also shown in Table 3. Individuals with COPD reported higher FOF with a median of 32 (24.75-36) compared to non-COPD individuals. There was no significant correlation between FEV1 and balance parameters in COPD patients (Table 4). 
TABLE 2: Comparison of gait parameters between COPD and Non-COPD subjects.

\begin{tabular}{|c|c|c|c|c|c|}
\hline \multirow{2}{*}{ Gait parameters } & \multicolumn{2}{|c|}{$\operatorname{COPD}(n=42)$} & \multicolumn{2}{|c|}{ Non-COPD $(n=45)$} & \multirow{2}{*}{$p$ value } \\
\hline & Median & Interquartile range & Median & Interquartile range & \\
\hline Step duration (msec) & 635.0 & $597.5,702$ & 610 & 515,735 & 0.60 \\
\hline Gait cycle duration (msec) & 1350 & $1195.0,1477.5$ & 1220 & 1120,1365 & 0.10 \\
\hline Swing duration (msec) & 1305 & 1220,1385 & 1230 & 1110,1350 & 0.044 \\
\hline Step length $(\mathrm{mm})$ & 545 & 500,621 & 523 & 456,593 & 0.23 \\
\hline Gait cycle length (mm) & 960 & $904.5,1087.7$ & 950 & 832,1054 & 0.24 \\
\hline
\end{tabular}

TABLE 3: Comparison of static and dynamic balance between COPD and Non-COPD individuals.

\begin{tabular}{|c|c|c|c|c|c|}
\hline \multirow{2}{*}{ Posturography parameters* } & \multicolumn{2}{|c|}{$\operatorname{COPD}(n=42)$} & \multicolumn{2}{|c|}{ Non-COPD $(n=45)$} & \multirow{2}{*}{$p$ value } \\
\hline & Median & Interquartile range & Median & Interquartile range & \\
\hline \multicolumn{6}{|l|}{ Static balance } \\
\hline NSEC-AP (mm/sec) & 11.1 & $6.8,16.7$ & 7.1 & $5.2,9.5$ & 0.002 \\
\hline NSEC-ML (mm/sec) & 7.0 & $4.8,9.3$ & 4.4 & $2.7,6.95$ & $<0.001$ \\
\hline NSEC-VM $\left(\mathrm{mm}^{2} / \mathrm{s}\right)$ & 12.9 & $11.8,20.6$ & 11.2 & $6.5,20$ & 0.11 \\
\hline NSEO-AP (mm/sec) & 8.3 & $7.95,9.65$ & 7.1 & $4.70,8.10$ & 0.023 \\
\hline NSEO-ML (mm/sec) & 5.1 & $3.8,6.2$ & 4.5 & $4.0,5.8$ & 0.213 \\
\hline NSEO-VM $\left(\mathrm{mm}^{2} / \mathrm{s}\right)$ & 21.0 & $14.0,26.3$ & 9.9 & $5.8,19.1$ & 0.00964 \\
\hline \multicolumn{6}{|l|}{ Dynamic balance } \\
\hline Timed up and go test time (sec) & 13 & 12,16 & 12 & 10,12 & $<0.01$ \\
\hline \multicolumn{6}{|l|}{ Fear of falls } \\
\hline Falls Efficacy Scale score & 32 & $24.75-36$ & 24 & 19,25 & $<0.01$ \\
\hline
\end{tabular}

NSEC-AP: normal standing eye-closed anteroposterior sway velocity. NSEC-ML: normal standing eye-closed mediolateral sway velocity. NSEC-VM: normal standing eye-closed velocity moment. NSEO-AP: normal standing eye-open anteroposterior sway velocity. NSEO-ML: normal standing eye-open mediolateral sway velocity. NSEO-VM: normal standing eye-open velocity moment.

TABLE 4: Spearman's rank correlation coefficient between Fev1\% and balance parameter for COPD participants.

\begin{tabular}{lcc}
\hline Balance parameter & $r$ & $p$ value \\
\hline NSEC-AP $(\mathrm{mm} / \mathrm{sec})$ & 0.14 & 0.39 \\
NSEC-ML $(\mathrm{mm} / \mathrm{sec})$ & 0.15 & 0.92 \\
NSEC-VM $\left(\mathrm{mm}^{2} / \mathrm{s}\right)$ & 0.25 & 0.10 \\
NSEO-AP & 0.23 & 0.80 \\
NSEO-ML (mm/sec) & 0.20 & 0.73 \\
NSEO-VM & 0.38 & 0.65 \\
\hline
\end{tabular}

NSEC-AP: normal standing eye-closed anteroposterior sway velocity. NSEC-ML: normal standing eye-closed mediolateral sway velocity. NSECVM: normal standing eye-closed velocity moment. NSEO-AP: normal standing eye-open anteroposterior sway velocity. NSEO-ML: normal standing eye-open mediolateral sway velocity. NSEO-VM: normal standing eye-open velocity moment. No significant correlation observed between balance parameter and Fev1\%.

\section{Discussion}

The static, dynamic balance, gait parameters, and fear of falling were significantly different in COPD individuals compared to age- and gender-matched non-COPD individuals.

It was found that the swing time in COPD individuals was significantly affected. The findings are consistent with a study by Yantes et al. [20, 21] which noted biomechanical gait alterations in COPDs. Another study by Annegarn et al. [13] showed that subjects with COPD walked at a decreased speed, decreased cadence, and greater medium-lateral variability during 6MWT compared to healthy elderly subjects measured by triaxial accelerometers. From the clinical point of view, reduced physical activity in daily life, impaired muscle strength, altered breathing dynamics, and poor balance are the mostly likely causes for gait abnormalities in COPD individuals [20]. Decreased levels of physical activity result in decreased muscle fiber cross-sectional area and reduction in mitochondrial density leading to skeletal muscle dysfunction which is commonly seen in COPD individuals, and these dysfunctions in the muscular system may impact walking patterns $[8,21]$.

The static and dynamic balance in individuals with COPD was found to be significantly affected compared to non-COPD people. Posturography was used in the current study to evaluate static balance, a more objective measure in which individuals with COPD had impaired postural control in the eye-open, eye-closed condition compared to nonCOPD individuals with increased sway velocity in anteroposterior (AP) and mediolateral (ML) and increased velocity moment (VM). Higher sway velocity and velocity moment is a sign of poor balance [15]. Findings are in the line with Almeida et al. [22] which noted individuals with COPD present balance deficits compared to healthy individuals. This can be explained by the rise in respiratory demand that is prevalent in people with COPD as it can impair balance due to increased activation of the trunk muscle $[22,23]$. The results of the present study indicate that the balance in COPD individuals was not influenced by the severity of obstruction, as there was no significant correlation found between these two parameters statistically.

In our study, we observed that the TUG score is at the borderline in both groups. However, compared to non- 
COPD individuals, COPD individuals had increased timed up and go test scores, which may be due to reduced peripheral muscle strength leading to changes in dynamic balance [24]. These outcomes are in line with the study of Beauchamp et al. [25] and Chang et al. [26].

Fear of falling has been frequently identified as a risk factor for falls [27]. Fear of falls in COPD individuals was found to be greater than in non-COPD individuals in this study. In COPD individuals, the FES-I score was 32, indicating high concern about falling. Improved susceptibility to falls has been due to worsening of dyspnoea and poor healthrelated quality of life (HRQOL) [28].

The finding of this study illustrates the need to include the evaluation of static and dynamic balance, gait, and risk or fear of falls in the systematic assessment of patients with COPD for pulmonary rehabilitation. Future studies can focus on the study of the impact of a comprehensive rehabilitation programme, including balance training on functional outcomes and the quality of life associated with health in patients with COPD.

\section{Conclusion}

In the present study, individuals with COPD showed reduced swing time, reduced static and dynamic balance, and greater fear of falls than age- and gender-matched individuals with no history of COPD. These results will help to explain gait dynamics, balance, and fear of falls in people with COPD. Future studies should focus on investigating the impact of muscle fatigue, physical activity on gait dynamics, and postural control in individuals with COPD.

\section{Data Availability}

The data used to support the findings of this study are available from the corresponding author upon request.

\section{Disclosure}

The part of the findings of this study were presented by the authors in European Respiratory Society conference.

\section{Conflicts of Interest}

The authors declare that they have no conflicts of interest.

\section{References}

[1] S. A. Quaderi and J. R. Hurst, "The unmet global burden of COPD," Global Health, Epidemiology and Genomics, vol. 3, 2018.

[2] R. Lozano, "Global and regional mortality from 235 causes of death for 20 age groups in 1990 and 2010: a systematic analysis for the Global Burden of Disease Study 2010," The Lancet, vol. 380, pp. 2095-2128, 2012.

[3] 2020, https://www.who.int/news-room/fact-sheets/detail/ chronic-obstructive-pulmonary-disease(copd)\#:\%7E:text $=$ The $\% 20$ Global $\% 20$ Burden $\% 20$ of $\% 20$ Disease,in $\% 20$ low $\% 20$ and $\% 20$ middleincome $\% 20$ countries.
[4] S. K. Jindal, "Emergence of chronic obstructive pulmonary disease as an epidemic in India," Indian Journal of Medical Research, vol. 124, pp. 619-630, 2006.

[5] S. K. Jindal and V. K. Vijayan, World Clinics: Pulmonary \& Critical Care Medicine-Chronic Obstructive Pulmonary Disease, JP Medical Ltd., Coimbatore, India, 2013.

[6] N. Cielen, K. Maes, and G. Gayan-Ramirez, "Musculoskeletal disorders in chronic obstructive pulmonary disease," BioMed Research International, vol. 2014, Article ID 965764, 17 pages, 2014.

[7] S. Mathur, D. Brooks, and C. R. Carvalho, "Structural alterations of skeletal muscle in copd," Frontiers in Physiology, vol. 5, p. 104, 2014.

[8] C. Ho, M. Mofarrahi, and S. N. Hussain, "Skeletal muscle dysfunction in patients with COPD," International Journal of COPD, vol. 3, no. 4, pp. 637-658, 2008.

[9] S. J. Butcher, J. M. Meshke, and M. S. Sheppard, "Reductions in functional balance, coordination, and mobility measures among patients with stable chronic obstructive pulmonary disease," Journal of Cardiopulmonary Rehabilitation, vol. 24, no. 4, pp. 274-280, 2004.

[10] A. Bueno-Cavanillas, F. Padilla-Ruiz, J. J. Jiménez-Moleón, C. A. Peinado-Alonso, and R. Gálvez-Vargas, "Risk factors in falls among the elderly according to extrinsic and intrinsic precipitating causes," European Journal of Epidemiology, vol. 16, no. 9, pp. 849-859, 2000.

[11] D. A. Lawlor, R. Patel, and S. Ebrahim, "Association between falls in elderly women and chronic diseases and drug use: cross sectional study," BMJ, vol. 327, 2003.

[12] A. D. Kuo and J. M. Donelan, "Dynamic principles of gait and their clinical implications," Physical Therapy, vol. 90, pp. 157-174, 2010.

[13] J. Annegarn, M. A. Spruit, H. H. Savelberg et al., "Differences in walking pattern during 6-min walk test between patients with COPD and healthy subjects," PLoS One, vol. 7, no. 5, Article ID e37329, 2012.

[14] P. Ramachandra, A. G. Maiya, and P. Kumar, "Test-retest reliability of the win-track platform in analyzing the gait parameters and plantar pressures during barefoot walking in healthy adults," Foot \& Ankle Specialist, vol. 5, no. 5, p. 306, 2012.

[15] S. Saxena, B. K. Rao, and S. Kumaran, "Analysis of postural stability in children with cerebral palsy and children with typical development," Pediatric Physical Therapy, vol. 26, no. 3, pp. 325-330, 2014.

[16] H. Ha, K. Cho, and W. Lee, "Reliability of the good balance system for postural sway measurement in poststroke patients," Journal of Physical Therapy Science, vol. 26, no. 1, pp. 121-124, 2014.

[17] D. Podsiadlo and S. Richardson, "The timed "Up \& Go": a test of basic functional mobility for frail elderly persons," Journal of the American Geriatrics Society, vol. 39, pp. 142-148, 1991.

[18] K. Okumiya, K. Matsubayashi, T. Nakamura et al., "The timed "Up \& Go" test is a useful predictor of falls in communitydwelling older people," Journal of the American Geriatrics Society, vol. 46, pp. 928-930, 1998.

[19] L. Yardley, N. Bayer, and K. Hauer, "Development and validation of falls efficacy scale-international (FES-I)," Age and Ageing, vol. 34, pp. 614-619, 2005.

[20] J. M. Yentes, H. Sayles, J. Meza, D. M. Mannino, S. I. Rennard, and N. Stergiou, "Walking abnormalities are associated with COPD: an investigation of the NHANES III dataset," Respiratory Medicine, vol. 105, no. 1, pp. 80-87, 2011. 
[21] J. M. Yentes, K. K. Schmid, D. Blanke, D. J. Romberger, S. I. Rennard, and N. Stergiou, "Gait mechanics in patients with chronic obstructive pulmonary disease," Respiratory Research, vol. 16, no. 1, p. 31, 2015.

[22] C. N. S. Almeida, R. A. d. Costa, K. P. Manso et al., "Static balance in older adults with chronic obstructive pulmonary disease undergoing pulmonary rehabilitation," Geriatrics, Gerontology and Aging, vol. 14, no. 2, pp. 98-107, 2020.

[23] M. D. Smith, A. T. Chang, and P. W. Hodges, "Balance recovery is compromised and trunk muscle activity is increased in chronic obstructive pulmonary disease," Gait \& Posture, vol. 43, pp. 101-107, 2016.

[24] M. K. Beauchamp, D. Brooks, and R. S. Goldstein, "Deficits in postural control in individuals with COPD - emerging evidence for an important secondary impairment," Multidisciplinary Respiratory Medicine, vol. 5, no. 6, p. 417, 2010.

[25] M. K. Beauchamp, K. Hill, R. S. Goldstein, T. Janaudis-Ferreira, and D. Brooks, "Impairments in balance discriminate fallers from non-fallers in COPD," Respiratory Medicine, vol. 103, no. 12, pp. 1885-1891, 2009.

[26] A. T. Chang, H. Seale, J. Walsh, and S. G. Brauer, "Static balance is affected following an exercise task in chronic obstructive pulmonary disease," Journal of Cardiopulmonary Rehabilitation and Prevention, vol. 28, no. 2, pp. 142-145, 2008.

[27] C. C. Oliveira, J. McGinley, A. L. Lee, L. B. Irving, and L. Denehy, "Fear of falling in people with chronic obstructive pulmonary disease," Respiratory Medicine, vol. 109, no. 4, pp. 483-489, 2015.

[28] M. Roig, J. J. Eng, D. L. MacIntyre et al., "Falls in people with chronic obstructive pulmonary disease: an observational cohort study," Respiratory Medicine, vol. 105, no. 3, pp. 461-469, 2011. 